\title{
Politique de la rue et politique des urnes
}

\author{
Albert Ogien \\ Centre détude des mouvements sociaux, Institut Marcel Mauss \\ Correspondence Address: 190-198 Avenue de France, Paris, France 75013 \\ Email: albert.ogien@ehess.fr
}

\begin{abstract}
The "Arab Spring" uprisings have unexpectedly led to a strikingly fast and worldwide movement of opposition to governments and economic powers. This sudden and unpredictable outbreak of protest has given birth to a new form of political action, which may be called "gatherings", i.e. people taking to the streets and occupying squares to claim a radical change of the political order through demands for a better or renewed democracy. Gatherings are innovative as they arise outside traditional ways of expressing political grievances (i.e. through parties, trade unions, NGOs and associations), have neither leader nor program, advocate non violence and disavow the system of representative government. This new way to practice street politics opposes ballot box politics as it claims direct democracy (general assemblies, open meetings, no decision by a majority, equally shared responsibilities, transparency, etc.) while ardently endorsing non violence. This commitment is contentious: how can one pretend toppling the rule of the rich and the powerful who benefit from an entrenched system of domination without making use of violence to oust them from their privileged position? This article aims at clarifying the terms of this question by exploring the way resorting to violence has been debated in many of these gatherings.
\end{abstract}

Keywords: democracy, direct democracy, critique of representative government, gatherings, street politics, non-violence.

La rue est redevenue, d'une manière spectaculaire et originale, un lieu politique majeur avec l'effervescence démocratique qui a saisi le monde depuis 2011. Traditionnellement, ce lieu connaît deux usages : accueillir des manifestations ou être le théâtre d'émeutes. En quelques mois, rassemblements et occupations de places ont inventé une autre manière d'intégrer la rue à une forme d'action politique. Chacun de ces trois usages à des caractéristiques différentes.

La manifestation est, en démocratie, un droit reconnu et garanti - même si des réglementations de plus en plus drastiques tendent à y restreindre la liberté de réunion et à encadrer les modalités de son expression. Dans les régimes autoritaires, elle est une mobilisation qui est soit orchestrée pour marquer le soutien au pouvoir, soit tolérée sous certaines conditions et dans certaines circonstances lorsquelle entend sopposer au régime, soit impitoyablement empêchée ou réprimée lorsquelle enfreint l'interdiction de faire entendre une voix dissidente.

Lémeute, elle, est simplement inacceptable sous quelque régime que ce soit; et, au nom du maintien de l'ordre, de la préservation de la paix civile et de la sauvegarde des biens privés, elle est systématiquement matée par les forces de lordre, le plus souvent avec

\footnotetext{
(c) Ogien A., 2014

(C) Centre for Fundamental Sociology, 2014
} 
l'assentiment ou le soulagement de la population (tant que l'intervention de la police ou de l’armée reste dans les limites du raisonnable). Que ce soit en régime démocratique ou autoritaire, l'émeute peut donner lieu à des négociations entre le pouvoir et des représentants ou des porte-parole des révoltés afin que le premier apporte une réponse aux griefs qui ont motivé le recours à la violence des seconds. C'est qu'il paraît évident aux deux parties que, si les conditions qui ont engendré l'explosion ne changent pas, le risque d'une nouvelle flambée continuera à planer. Mais l'ouverture de ces négociations ne peut se produire qu'une fois le calme revenu et l'ordre rétabli - sans que rien n'assure que les conditions qui ont suscité la violence seront effectivement modifiées.

Quant aux rassemblements et aux occupations, leur statut politique est encore indécis. Car bien que l'installation en masse sur une place publique et l'organisation d'un campement soient des actes illégaux qui, en tant que tels, pourraient être immédiatement sanctionnés, la décision de faire procéder à leur dispersion a généralement été suspendue. Une des raisons qui ont dissuadé les pouvoirs d'intervenir est le fait que ces mouvements ont, dès leur origine, ostensiblement affiché leur engagement en faveur de la non violence. Cette résolution, qui s'est confirmée par les actes, leur vaut une légitimité conditionnelle et provisoire : rassemblements et occupations sont toujours restés sous la menace du démantèlement et ont dépendu du bon vouloir des pouvoirs publics, qui guettaient le moment opportun pour renoncer à cette tolérance et éradiquer cet abcès de contestation de la loi. Une autre raison de cet attentisme est toute matérielle : le nombre subit d'occupants et la soudaineté de leur installation sur les places a pris tout le monde par surprise. Confronté à un débordement aussi inattendu, un pouvoir peut hésiter devant le coût financier, humain et politique que représentent un assaut et une éviction de force devant des caméras friandes d'images choc. Un troisième facteur a joué dans la décision de surseoir à une intervention : le soutien que ces mouvements ont souvent reçu de la part de la population, dont les sondages dopinion montraient quelle tenait la protestation pour juste. Un dernier élément est venu calmer les ardeurs répressives des autorités : ce nouvel usage politique de la rue oblige ses organisateurs à assurer la vie paisible d'un campement. Ce qui les conduit à entamer et poursuivre des pourparlers avec les riverains et les pouvoirs locaux et à instaurer des rapports de coopération avec la police. La durée même de ces installations a été rendue possible par le fait que leurs responsables ont fait la preuve quotidienne qu'ils étaient capables de prendre en charge les questions d'approvisionnement, de sécurité et d'hygiène qui incombent, en temps normal, aux services spécialisés. Et tant que lordre régnait sur les places, sous le regard de la police, celle-ci ne disposait d'aucun prétexte justifiant l'urgence d'une action répressive.

Les rassemblements et les occupations se sont ainsi imposés comme des formes d'action politique légitimes, avec cette caractéristique paradoxale d'être, comme les émeutes, des actes d'illégalité et, comme les manifestations, un mode de protestation non violent. Et cette légitimité a pu se maintenir près d'un mois durant. Mais la légitimité n'est pas une condition de l'efficacité : quel peut être l'impact d'une forme d'action politique qui affiche sa volonté de préserver sa nature non violente? 
Les rassemblements et les occupations ne sont ni un happening, ni un festival de musique : ce sont des mouvements de protestation. Ce qui veut dire que ceux qui s'y sont engagés l'ont fait, en partie au moins, au nom d'une revendication à laquelle ils ont espérés obtenir satisfaction. Cet espoir, qui confère sa nature politique à l'action, crée une attente et conduit les participants à porter un jugement sur l'utilité de leur engagement ; d'autant plus qu'il peut devenir éprouvant, dangereux et fatigant. Un des critères utilisés pour former ce jugement est le succès ou les acquis que la mobilisation a permis d'engranger. Or c'est bien ce qui est le plus difficile à établir : une fois passé le temps de la mise en place d'un ordre local et des conditions de sa reproduction au quotidien, une fois passées l'exaltation des premières assemblées et la répartition des tâches en diverses commissions, une fois les responsabilités distribuées et les règles de conduites collectives édictées, une fois l'infrastructure de communication installée, une fois les débats enthousiasmants devenus répétitifs ou lassants, arrive un moment où il faut dresser le constat de ce à quoi tout cela peut servir. Si le sentiment prévaut alors que rien n’a changé (ce qui est généralement le cas) et que rien ne changera vraiment, une interrogation saisit les participants : doit-on renoncer (en admettant éventuellement que l'existence du mouvement était, à soi-seul, le but de l'action) ou décider d’opérations qui ont une autre virulence ? Et c'est bien ce qui s'est produit à mesure que l'épuisement gagnait les places, à la Puerta del Sol ou à Zuccotti Park comme à Tahrir ou à Maïdan. Et à la question : " pourquoi tenir la place ?", la réponse n'a pas été (c’est le moins qu’on puisse dire) la même dans tous les lieux où elle s'est posée.

Dans les situations démocratiques, comme à Madrid ou à New York, l'idée d'abandonner la non-violence a été émise et débattue au bout de trois semaines d'occupation environ. Et les arguments qui se sont opposés dans ce débat ont retrouvé les lignes d'un clivage classique. Pour les uns, se faire réellement entendre des gouvernements et des puissants réclamait désormais de recourir à des actions menaçant directement et matériellement leur pouvoir ; pour d'autres, la non-violence était la seule garantie de l'enracinement et du développement d'un mouvement dont l'objet n'avait jamais été d'entrer dans un rapport de force avec les autorités, mais de faire entendre la voix ignorée des citoyens et d'exiger un changement radical dans la manière dont les affaires publiques sont conduites. La question du recours à la violence n’a pas été tranchée par les assemblées, mais la décision de lever le camp fut prise peu après qu'elle ait été publiquement posée à Madrid (au bout de quatre semaines d'occupation) ; tout comme celle d'évacuer le Park fut proclamée par les tribunaux et exécuté, sans opposition, par la police à New York (après deux mois d'occupation). À Londres, loccupation dura près de cinq mois, sur un terrain gracieusement mis à disposition par l'Église anglicane, et s'arrêta volontairement et dans la lassitude, comme ce fut également le cas à Genève, Amsterdam ou Francfort.

Dans les situations autoritaires, comme au Caire ou à Kiev, la question de la non-violence a donné lieu à tout autre traitement. C'est sans doute que le contenu de la revendication qui a motivé le mouvement y a pris une dimension plus vitale et plus ciblée : la chute d'un régime de corruption et de terreur. Dans ces cas, loccupation a une vocation différente : entrer dans une confrontation pacifique mais irrémissible avec des dirigeants 
désavoués. Et tant que le pouvoir ne cède pas, l'alternative est claire : dispersion par la force armée (sur le mode de la répression du campement de la place Tienanmen à Pékin en 1989) ou démission ou fuite des dirigeants (Ben Ali en Tunisie, Moubarak puis Morsi en Egypte, ou Ianoukovitch en Ukraine). Ici, la question de la non violence se pose en d'autres termes : dans la mesure où la majorité des participants refuse de renoncer avant d'avoir obtenu satisfaction, recourir à la violence apparaît comme un moyen dont il pourrait être nécessaire de se servir, le cas échéant, pour mettre en application la résolution collective portée alors par une maxime : vaincre ou mourir.

Le déroulement de ces événements illustre un phénomène : une sorte de logique de passage de la non violence à la violence s'enclenche dès que ceux qui mènent un combat politique pour une cause qui est essentielle à leurs yeux voient que les cibles qu'ils se donnent se dérobent devant eux ou ne répondent pas aux attaques dont ils font l'objet. On sait que l'idée de renoncer à une action politique faute de réaction et sans que rien n'ait été obtenu est, généralement, coûteuse au sens où elle dévalorise les efforts qui ont été consentis pour faire aboutir une revendication que l'on croit juste et créent un sentiment d'échec qui affaiblit la possibilité de relancer une mobilisation. Mais le sens commun politique le dit : il faut savoir arrêter une grève... et un rassemblement ou une occupation aussi. Ces formes d'action politique ne sont pas faites pour durer éternellement. Il n'est pas rare cependant d'observer que, lorsqu'elles cessent, reste toujours un groupe pour lequel ce renoncement sonne comme une trahison et qui appelle à poursuivre la lutte. Puis il peut arriver que quelques uns se résolvent à passer aux choses sérieuses en décidant de se livrer à des actes de violence, voire à s'engager dans le terrorisme. Le fait que ce choix ne concerne jamais qu'une infime minorité d'individus est une indication de l'existence d'une sorte de préférence pour la non violence ; ou, plus simplement, celle de la peur face à l'incertitude, aux périls ou au chaos que la violence ne manque pas de déchaîner ; ou encore la certitude que ce genre d'action ne parviendra pas à altérer l'inflexibilité des pouvoirs attaqués. C'est cette question du rapport entre violence et politique que les débats qui ont eu lieu dans les rassemblements et les occupations ont eu à affronter et à résoudre de façon pratique.

\section{De la violence en politique}

La conception que nous nous faisons du rapport entre violence et politique reste fixée par la définition que Weber a donnée de l'Etat en tant que "détenteur du monopole de la violence légitime". Cette définition trace une frontière : l'Etat a mission de garantir la paix civile et les interventions qu'il doit mener pour le faire sont, tant qu'elles demeurent épisodiques et que la légitimité de son pouvoir n’est pas sapée par cette répression, relativement incontestables. On sait que, chez Weber, cette légitimité peut avoir trois fondements : la tradition, le charisme ou la rationalité administrativo-bureaucratique - qui est le régime de légitimité qui prévaut dans les démocraties contemporaines où la détention du pouvoir procède du peuple, dans la représentation qu'il donne de lui-même à loccasion d'élections. Certains considèrent cependant que cette représentation est faussée puisque 
les institutions d'Etat (école, justice, police, travail social, etc.) se chargent de reproduire les conditions de la domination des puissants, tout en s'arrangeant pour contrôler les forces qui viendraient la contester ou la remettre en cause. Dans cet arrangement, les actions menées par ceux qui dénoncent la violence de l'ordre établi tombent sous la qualification officielle de "violence" alors que celles qui les répriment (c'est-à-dire le travail de la police, des services secrets ou des officines qui traquent ou font taire les contestataires les plus virulents ou les plus dangereux) se nomment opérations de rétablissement de la sécurité publique. Mais cette distinction entre usage légitime et illégitime de la violence se réduit-elle uniquement à la question du monopole de son exercice au service de l'imposition d'une idéologie visant à favoriser la reconduction d'une hégémonie de pouvoir? Ne serait-elle pas également liée au processus de pacification des relations politiques qui caractérise les régimes démocratiques?

On mesure généralement la vitalité d'une démocratie à l'aide d'une série d'indicateurs : élections libres et sincères ; séparation réelle des pouvoirs ; organisation de la société civile ; liberté d'information, d'association et de contestation ; impartialité de la justice ; garantie du droit à la défense ; respect des droits de l'homme et du droit de propriété ; abolition du délit d’outrage ; intervention des citoyens ordinaires dans le processus de décision législative. S’il n’existe pas de régime démocratique si parfait qu’il remplirait pleinement l'ensemble de ces indicateurs, on peut dire qu'un Etat qui prétend établir un tel régime s'inscrit dans une dynamique qui tend à les remplir.

Deux autres indicateurs permettent cependant d'apprécier l'intensité d'une vie démocratique : l'extension accordée à la négociation et au droit dans la résolution des conflits et le degré de pacification des rapports politiques. Il est en effet de la nature du processus de démocratisation d'installer une habitude de consensus, qui contribue à réduire les antagonismes et finit par produire un assentiment des citoyens pour exclure le recours à la violence physique des formes acceptables d'expression des opinions discordantes. De façon paradoxale, cet assentiment inocule lentement les germes du désintérêt et de l'indifférence pour les affaires politiques, dans la mesure où légalisation des conditions se poursuit, en réduisant les raisons d'un désaccord fondamental. C'est un peu cela qui explique la convergence des programmes des partis et, conséquemment, l'augmentation du taux d'abstention aux élections. D’une certaine manière, plus le processus dure sans être remis en cause (par une crise grave, une révolte alarmante, un coup d'Etat ou une guerre), plus il établit la légitimité d'une même modalité d'exercice du pouvoir, admise par tous les partis qui obtiennent, alternativement, la responsabilité de la conduite des affaires publiques (avec de modestes variations à la marge). Dans ce lent mouvement d'apaisement des passions politiques, les citoyens en arrivent tout logiquement à considérer que les enjeux électoraux ne sont plus vraiment déterminants, et le principe de l'alternance semble lui-même frappé d’obsolescence. Avec cette atténuation de la virulence de la compétition inhérente au système représentatif, la possibilité de remettre radicalement en cause les règles instituées de la vie politique semble se vider de toute substance. Mais alors, où aller trouver les ressources permettant de s'opposer à la violence de l'Etat qui, elle, continue 
à s'exercer, et parfois sans ménagements pour ceux dont les gouvernants refusent d'entendre les doléances ou jugent quelles sont irrationnelles?

En fait, l'exclusion de la violence des relations politiques en démocratie est toujours conditionnelle. Rien nempêche jamais des citoyens d'y recourir pour manifester leur désapprobation de lordre courant des choses. Il peut ne s'agir que d'une éruption d'exaspération ou de colère (vandalisme, pillages ou émeutes) motivée par des conditions d'existence devenues insupportables et clamant, par ce truchement, un désir de les voir transformées. Les suites données à ces éruptions varient avec le degré de développement politique du pays concerné : les réponses apportées aux émeutes de la faim ou de la colère dans les pays pauvres ne sont pas les mêmes que celles qui le sont aux émeutes urbaines qui éclatent dans les quartiers de relégation des pays développés. Parfois cependant, des groupements politiques se constituent autour d'un projet qui tient le recours à la violence pour moyen d'expression exclusif d'une revendication qui vise le renversement d'un pouvoir en place et la destruction du mode de domination qu'il impose (révolte armée, terrorisme, guérilla, ou révolution).

En règle générale, c’est la répugnance obstinée des dominants à tenir compte des exigences légitimes des dominés (en termes de dignité, de justice, dégalité ou de vie décente, etc.) qui justifie la nécessité d'un usage direct de la violence. Mais si la lutte armée semble légitime lorsqu'il s'agit de renverser un régime autoritaire ou un pouvoir qui bafoue délibérément les libertés fondamentales, elle ne l'est en aucun cas dans un Etat de droit. Dans ce cadre, un principe domine : force doit rester à la loi dans la mesure où elle est lémanation de la souveraineté populaire et que rien n'interdit, en principe, quelle puisse être modifiée à l'occasion d'une alternance. C'est en ce sens que la violence est conçue comme antinomique de la démocratie. Pourtant, la manière dont la question du passage à la violence s'est trouvée posée à l'occasion des rassemblements et occupations conduit à appréhender cette antinomie de façon plus dynamique et à se demander à quelles conditions et dans quelles circonstances l'expression d'une revendication politique en vient-elle à envisager le recours à la violence.

\section{Le spectre des usages de la violence}

Linstitution d'un Etat de droit néradique pas complètement l'usage de la violence de la vie politique, mais lui assigne une place résiduelle dans les changements qui l'affectent. C'est en tout cas la leçon qu'il faut tirer des faits. D'une part, on observe régulièrement que des manifestations violentes, des saccages, des caillassages ou des destructions viennent quelquefois exprimer, en démocratie, la force d'une revendication catégorielle (frondes paysannes, jacqueries, occupations d'usines, destruction de champs d'OGM, démontage d'établissements de restauration rapide, etc.). D’autre part, et de façon moins dramatique, on sait que des figures pacifiées de violence apparaissent dans l'espace public, comme c'est le cas lorsqu'une organisation politique s'engage dans la "construction d'un rapport de forces". Ce qui apparente cette action à la violence est le fait qu'elle pose en principe un antagonisme absolu entre parties en conflit, conteste la possibilité d'un consensus et rejette 
la volonté d'y parvenir. Et cette modalité pacifiée de confrontation brutale peut prendre des allures extrêmes : celles de la "grève générale" ou d'une grève de la faim

Ces formes ritualisées d'usage de la violence sont relativement bien intégrées dans lordre courant des choses démocratiques. Leur fonction est connue : faire constamment peser une menace sur une négociation, en brandissant le spectre d'une explosion ou d'un acte irréparable, qui ne se produisent que de façon exceptionnelle. Pour illustrer ce phénomène, on peut considérer une action politique - par exemple la mobilisation pour l'obtention de cartes de séjour pour des travailleurs devenus clandestins du fait d'un durcissement de la loi ou du zèle d'un directeur de service. Pour ceux qui embrassent cette cause, cette revendication est juste et légitime, ce que leur confirme le fait qu'elle est publiquement défendue par des syndicats, des associations ou des comités de soutien. Imaginons que des négociations souvrent avec les pouvoirs publics et que ceux-ci se montrent inflexibles. La lutte monte d'un cran : les travailleurs occupent des locaux et décident d'arrêter de s'alimenter jusquà obtenir satisfaction. Imaginons encore que les autorités réagissent en faisant évacuer les locaux et en nourrissant de force ceux qui persistent à ne pas vouloir manger. Faut-il alors renoncer (ou accepter de négocier au cas par cas de façon cachée l'attribution de cartes de séjour pour que le gouvernement ne perde pas la face) ou envisager de passer à la violence (que les participants peuvent parfois retourner contre eux-mêmes, en se suicidant ou en s'immolant par le feu en un geste altruiste ou simplement désespéré) ?

Un autre exemple de cette interrogation qui naît, dans le cours même du combat politique, au sujet du bien-fondé du recours à la violence est fourni par Jérome Lindon dans son engagement déditeur pour dénoncer et faire cesser la pratique de la torture durant la guerre d'Algérie. Il commet, tout d'abord, un acte de désobéissance civile en publiant délibérément deux textes décrivant les exactions commises en France et en Algérie : Pour Djamila Bouhireb, de George Arnaud et Jacques Vergès, et La question de Henri Alleg qui est saisi le 27 mars 1958. Il récidive en publiant L’Affaire Audin de Pierre Vidal-Naquet, qui prône l'insoumission et le refus d'obéir à des ordres manifestement illégitimes, ce qui tombe sous le coup de la loi. Le 18 juin 1959, Lindon publie la Gangrène, cinq témoignages détudiants algériens torturés par la DST, qui est immédiatement saisi (mais dont la réédition en juillet 1959 ne le sera pas). En continuant à publier des ouvrages dont le caractère subversif est revendiqué, Lindon vise ostensiblement à être mis en accusation devant un tribunal, aux seules fins de porter la question de la torture dans le débat public. Or, si les livres qu'il édite sont régulièrement saisis dans le but de ruiner sa maison dédition, un seul procès lui sera intenté, en décembre 1961, pour "incitation de militaires à la désobéissance" à loccasion de la publication d'un roman intitulé Le Déserteur, écrit par un officier ayant participé à cette guerre et défendant la légitimité de la désertion. L'interdiction du livre sera confirmée par le tribunal, et Lindon condamné à une amende qui sera réglée par un groupe déditeurs en soutien aux Editions de Minuit. Mais, comparaissant plus tard devant un tribunal comme témoin dans le procès d'un groupe de militants venant en aide au FLN (Front de Libération Nationale algérien) — le réseau Jeanson —, Lindon déclarera, comme pour défendre l'action de ces "porteurs de valises" qui, en se mettant au 
service de la résistance algérienne en transportant de l'argent pour acheter des armes, ont choisi de commettre des actes qui, à lépoque, relevaient de la trahison :
"au cours de ces trois dernières années, les réactions provoquées par ces ouvrages sont pratiquement nulles $\mathrm{Ni}$ les auteurs de ces textes, ni moi-même n'avons jamais été poursuivis en diffamation. Ce qui est plus grave, c'est que, à ma connaissance, aucun des faits dénoncés dans ces ouvrages n’a été, pour leurs auteurs, une cause d'inquiétude [...] Quand je vois l'inefficacité de la lutte que j’ai menée; que d'autres ont mené avec moi pour une cause qui est évidemment légitime, celle de la lutte contre la torture, quand je vois que cette lutte a été totalement inefficace, je suis obligé de me dire que c'est peut-être parce qu'elle est restée dans le strict domaine de la légalité."

Faire de l'usage direct de la violence le moyen de l'action politique n'est toutefois pas une démarche courante. Elle a longtemps été l’apanage de nationalistes luttant pour la libération et l'indépendance de leur pays ; ou de révolutionnaires visant à détruire le système d'exploitation capitaliste. Plus récemment, elle est celle de militants d'extrême-droite voués à la défense de la civilisation occidentale menacée par l'invasion islamiste" (pour lesquels le meurtrier norvégien Anton Breivik est devenu une icône) ; ou celle d'activistes anarchistes, altermondialistes ou écologistes en lutte contre l'exploitation à leurs yeux éhontée et criminelle des êtres humains et des ressources naturelles par un système productif exclusivement mû par le profit à court terme. La cause écologiste introduit une différence : alors que les révolutionnaires définissent un ennemi, le combat pour l'environnement s'attaque à la somme des atteintes à la planète et à la biosphère, qu'elles soient celles des entreprises ou celles de individus, indépendamment du lieu où elles se produisent. De ce fait, s'en prendre violemment à des activités ou des industries qui contribuent, dans un cadre national, à dégrader l'environnement ne suffit en rien pour gagner ce combat. Ce sont tous les Etats du monde qui devraient se plier à une même discipline, en leur faisant adopter des mesures drastiques de prévention et de sécurité. La chose n’est pas simple à réaliser - même si des organisations internationales œuvrent à essayer de la faire advenir. En dépit de cette limite, les activistes qui défendent l'avenir de la vie sur la planète peuvent s'en prendre, de façon violente, à des agissements qui, dans leur pays de résidence, persistent à porter atteinte à l'air, à l'eau, au climat, aux espèces animales et végétales, en ignorant délibérément les dégâts qu'ils causent. C’est le cas, en particulier, des activistes écologistes radicaux américains qui ont choisi le terrorisme pour sauver la nature et l'humanité de son sort fatal.

En somme, de son usage direct à ses usages pacifiés en passant par ses usages sporadiques et son usage légitime à des fins de maintien de lordre social, la violence ne cesse de figurer l'arrière-plan (redouté ou intégré) sur lequel se détache l'action politique. C’est pourquoi il est possible d'affirmer que la croyance en l'efficace de la non violence est non seulement infondée, mais quelle conforte la distinction entre violence légitime et illégitime qu'impose un ordre de domination établi. 


\section{La rationalité politique de la non violence}

La critique du choix de la non violence en politique renvoie à une question : l'activité politique sert-elle à aménager la hiérarchie et l'inégalité des conditions des citoyens tout en préservant un mode de vie dont l'injustice ou le caractère insoutenable est avéré ; ou bien consiste-t-elle à mettre un terme à cet état de fait en imposant une véritable égalité, la fin de la domination et de l'exploitation des humains comme de la nature ? Deux options s'affrontent sur ce point - qui ont alimenté les débats qui se sont tenus dans les rassemblements et les occupations : d'un côté, les réalistes admettent qu'il faut prendre les choses telles quelles sont et composer avec elles du mieux possible ; de l'autre, ceux qui affirment que ce qui passe pour être la réalité est un leurre qui masque les intérêts de quelques uns et qu'il faut en dévoiler le caractère trompeur ou aliénant - ce qui doit probablement impliquer le recours à la violence. Cette ligne de partage sépare également deux manières de mener un combat pour le changement : soit agir dans lordre du politique, c'est-à-dire dans ces multiples arènes publiques dans lesquelles un travail saccomplit qui fait advenir des problèmes d'intérêt collectif et définit les limites du bien commun comme la manière de l'instaurer ; soit agir dans le monde de la politique, c'està-dire œuvrer au renversement d'un régime ou à la destitution d'une domination afin de permettre l'avènement de la liberté et de l'autonomie des individus. C'est du point de vue de la politique que le choix de la non violence peut être tenu pour irréaliste ou naï. Et il est vrai quon peut lui reprocher d'introduire un écart insurmontable entre le but poursuivi (modifier les effets de la stratification sociale et la répartition inégale des avantages qu'elle induit) et le moyen adopté pour l'atteindre (une forme d'action non violente). Car qui peut sincèrement croire que les milieux qui tirent bénéfice d'une domination ou d'un système d'exploitation renonceraient à certains de leurs privilèges contestés sans y être contraints par la force ? Mais ce jugement est partial : il ne prend pas en ligne de compte le fait que le choix de la non violence peut avoir un caractère stratégique. Comment donc ceux qui optent pour cette manière d'agir en politique conçoivent-ils son efficace?

Largumentation qu'utilisent les organisations politiques appelant à l'usage direct de la violence suit un raisonnement qui enchaîne une suite de propositions : les intérêts des groupes sociaux réunis au sein d'une même entité politique (qu'ils soient de classe, ethniques ou existentiels) sont totalement irréconciliables; d'où il découle qu'un pouvoir impose la domination sans partage d'un système qui sert des intérêts particuliers contraires à ceux de l'humanité ou de la planète ; et que le maintien de l'ordre établi vise uniquement à reproduire les conditions de l'injustice, de l'indignité et de l'indécence de certains de ces intérêts. Au final, c'est parce qu'elle ne prend pas acte de la nature foncièrement agonistique de la société que la non violence est tenue pour conforter un "consensus" qui, fondé sur la violence quexercent les institutions de la reproduction de la domination, profite aux puissants.

Dans ce raisonnement, la pacification des relations politiques se présente comme une illusion ou un leurre idéologique. Ce leurre est souvent présenté, comme le fait A. Brossat, en termes moraux : 
"Le mouvement de pacification de la vie sociale et du domaine politique a pour enjeu un formatage rigoureux des perceptions collectives de "la violence » et une réforme radicale du code destiné à séparer le violent du non-violent [...] Dans ces conditions, «la violence » tend à devenir d'une manière exclusive le fait de l'autre du pauvre, de l'immigré, de la plèbe mondiale, de l'islamiste, de l'Etat-voyou. Elle tend toujours davantage à faire l'objet de rites de détestation et d'exorcismes, à devenir une question morale plutôt que politique ou sociale. Son évocation péjorative devient un moyen de gouvernement des populations à la peur et à la sécurité, davantage qu’à la paix."

Avant d'admettre cette idée, on peut tout de même se demander comment la violence pourrait jamais être collectivement adoptée comme fondement d'un mode acceptable et viable de régulation des conflits interpersonnels. Pour penser cette éventualité, il suffit simplement de considérer la situation qui naît lorsque la loi du plus fort fait droit, comme c'est le cas lorsque l'injustice et la peur sont imposés par les pouvoirs despotiques ou les mafias ; lorsque la corruption généralisée ordonne les relations d'échange ; ou lorsque les bandes ou les caïds font régner l'ordre machiste de la "culture de rue" dans les quartiers de relégation. Que ce soit pour des raisons de sensibilité ou de principe, on peut admettre que la violence fait l'objet, dans les démocraties avancées en tout cas, d'une aversion et d'un rejet tant de la part du personnel politique que de celle des citoyens. La meilleure illustration de ce phénomène serait le discrédit qui y frappe, de façon rétrospective, les mouvements de type Action directe, Bande à Baader, Brigades rouges ou ETA ; ou, de façon plus contemporaine, les attentats terroristes. Le constat de l'aversion pour la violence est aussi celui que dressent les activistes les plus radicaux, lorsqu'ils admettent qu'ils sont voués à être minoritaires (les gens préfèrent l'ordre au chaos) ou préconisent le recours à une violence ciblée (concentrer les attaques contre des biens matériels ou symboliques et être attentifs à ne pas faire de victimes) pour ne pas provoquer le rejet de ceux qu'ils assurent défendre.

Les rassemblements et occupations de places ont fait le choix inverse : celui du strict respect de la non-violence. Et ce n'est ni par faiblesse, ni par aveuglement, ni par conformisme idéologique, mais pour répondre à une stratégie politique fondée en rationalité. La force de ces formes d'action, comme celle de tous les mouvements de protestation politique extra-institutionnelle, se trouve essentiellement dans la légitimité qu'ils parviennent à obtenir à la fois par la justesse de la revendication qu'ils expriment et par le nombre de personnes qu'ils arrivent à réunir pour la défendre. Une condition de leur succès réside donc dans un élément : l'unanimisme de la revendication, c'est-à-dire le fait qu'elle puisse valoir pour tout un chacun sans exclusive. Les meilleurs exemples de cet unanimisme se trouvent dans deux slogans qui ont été repris, à toutes sortes de fins, aux quatre coins de la planète depuis 2011 : “Nous sommes les 99\%” ou "Le peuple veut". On voit immédiatement le flou qui entoure ces invocations. Mais ce flou a la vertu cardinale d'exclure le moins de personnes possible de la participation, ce qui permet de remplir un impératif propre à cette forme d'action politique : faire nombre. Maintenir le caractère unanimiste de la revendication est ainsi la première arme dont il faut essayer de préserver 
la puissance. Une seconde raison stratégique qui plaide en faveur de la non violence est le souci de ne pas disqualifier la revendication en alimentant la propagande que les pouvoirs en place, via les médias, ne manqueraient pas de faire en montant en épingle des actes délictueux ou en insistant sur le caractère effrayant ou barbare de certains manquements à la bonne morale ou à l'hygiène. Une troisième raison est de ne pas rendre légitime la répression policière, que des actes de provocation et de violence viendraient instantanément justifier. Une autre raison du choix de la non violence vient d'une appréciation réaliste du rapport de force et de l'implausibilité d'un changement immédiat et brutal de la situation dont on conteste la justesse ; et l'un de ses corollaires : mettre au jour la violence du pouvoir en lui opposant le calme d'une voix qui s'exprime de façon résolue pour le droit et contre l'injustice, en acceptant même de se faire violenter sans réagir ou en offrant son corps désarmé à la répression. Une dernière raison du choix stratégique de la non violence est que le caractère pacifique de la protestation est un exemple qui permet d'œuvrer à une transformation des mentalités à long terme et à l'avènement d'un changement politique majeur.

Telles sont les raisons quon peut avancer pour convaincre du caractère réellement politique du choix de la non violence. Mais ce choix peut également être justifié en montrant qu'il a produit des effets aussi concrets que ceux que la violence aurait pu avoir. Les exemples abondent : Gandhi, Luther King, Walesa, Havel, Sakharov, Mandela, Aung San Suu Ky. A un moindre niveau, c'est aussi ce qu'on peut dire de la revendication de démocratie réelle telle qu'elle s'est exprimée dans les rassemblements et occupations de places. Elle a tout d'abord inscrit, dans le débat public et sur l'“agenda" de l'action politique officielle, de questions qui n'y figuraient pas. Elle a ensuite hâter les transformations de l'organisation et de la vie interne des partis et des syndicats, comme par exemple l'introduction de "primaires" dans les partis, le rajeunissement de leurs cadres, les nouvelles modalités d’adhésion dans les syndicats, et la modification des revendications pour faire droit à des éléments de contestation inédits. Elle a également fait valoir la nécessité de réformer les façons de faire de la politique, dénonçant l'opacité des décisions, la corruption des élites et la connivence entre professionnels de la politique et journalistes et rappelant à l'exigence de rendre au politique la noblesse de sa vocation originelle : être au service exclusif du bien commun. Elle a enfin entraîné une reconfiguration de l'“offre" politique. C’est ainsi qu'ont vu le jour ou se sont constitués des Forums (regroupant un ensemble de forces de contestation sociale, en particulier syndicats et associations, comme en Espagne), des "mouvements" (comme les M5S en Italie), des partis (Parti X en Espagne, M5S en Italie, Le Parti Pirate en Suède, Allemagne ou France) ; ailleurs, des porte-parole des occupations se sont résolus à rejoindre des listes de partis traditionnels (comme en Israël). La décision de certains d'accepter de prendre part active au processus électoral a souvent modifié les équilibres politiques traditionnels, en soulevant la perplexité des experts, des journalistes et des analystes (ré-élection de Barack Obama, succès du nouveau parti "Yesh Atid" - "Un avenir existe" - en Israël et du M5S en Italie, victoire de lopposition au Québec à la suite du Printemps érable). 
Tous ces développements démontrent que, plus une démocratie est avancée, plus une certaine continuité s'instaure entre l'ensemble des modalités d'expression des voix dissonantes des citoyens. Admettre une telle continuité oblige à reconnaître que la participation à la vie politique se construit, en démocratie, comme une sorte de bricolage, qui permet à chacun, sans contradiction, de recourir, selon les circonstances et de la manière dont il l'entend, à la procédure électorale (le vote ou l'abstention), à la négociation sociale (le droit du travail), à l'opposition politique (partis, syndicats, associations), à l'arme du droit (tribunaux ordinaires, juridictions spécialisées, instances européennes), à la manifestation et à la grève, à l'expression publique et artistique, à des formes d'action non-violente (désobéissance civile, rassemblements, occupations) ou à certains usages pacifiés de la violence. Ce qui veut dire que tout pouvoir qui veut faire progresser la démocratie doit pleinement accepter de voir se multiplier les formes daction politique que les citoyens adoptent pour défendre une de leurs revendications. Et cela à la seule condition que cette revendication se formule et s'exprime sur un mode tenu pour légitime par les membres de la société civile que cette revendication concerne, et pas par des autorités publiques ou religieuses qui l'autorisent ou la tolèrent.

\section{Bibliographie}

Bourdieu P., Passeron J.-C. (1970) La reproduction: eléments pour une théorie du système d'enseignement, Paris: Minuit.

Castel R. (1981) La gestion des risques, Paris: Minuit.

Hobsbawm E. (1972) Les bandits, Paris: Maspéro.

Foucault M. (1975) Surveiller et punir, Paris: Gallimard.

Laacher S., Terzi C. (2012). Comment faire peuple? Le cas des protestations publiques au Maghreb. L’Année du Maghreb, no 8, pp. 87-102.

Nez H. (2012) Délibérer au sein d'un mouvement social: ethnographie des assemblées d'Indignés à Madrid. Participations, vol. 4, no 3, pp. 79-102.

Pettit P. (2004) Républicanisme: une théorie de la liberté et du gouvernement, Paris, Gallimard.

Provocation à la désobéissance: le procès du "Déserteur", Paris: Minuit, 2011.

Roberts A., Ach T. G. (eds.) (2009) Civil Resistance and Power Politics: The Experience of Non-violent Action from Gandhi to the Present, Oxford: Oxford University Press.

Sémelin J. (2011) Résistance civile et totalitarisme, Bruxelles: André Versaille.

Simonin A. (2012) Le droit de désobéissance, Paris: Minuit.

Vakaloulis M. (2013) Précarisés, pas démotivés: les jeunes, le travail et l'engagement, Paris: La Dispute.

Weber M. (1971) Economie et société, Paris: Plon. 